\title{
Bending a Photonic Wire into a Ring
}

2 Henrik Gotfredsen, ${ }^{\dagger 1}$ Jie-Ren Deng, ${ }^{\dagger 1}$ Jeff Van Raden, ${ }^{1}$ Marcello Righetto, ${ }^{2}$ Janko Hergenhahn, ${ }^{1}$ Michael Clarke, ${ }^{3}$ Abigail Bellamy-Carter, ${ }^{3}$ Jack Hart, ${ }^{3}$ James O'Shea, ${ }^{3}$ Timothy D. W. Claridge, ${ }^{1}$ Fernanda Duarte, ${ }^{1}$ Alex Saywell, ${ }^{* 3}$ Laura M. Herz, ${ }^{* 2}$ and Harry L. Anderson*1

(† These authors contributed equally)

1. Department of Chemistry, University of Oxford, Chemistry Research Laboratory, Oxford OX1 3TA, UK

2. Department of Physics, University of Oxford, Clarendon Laboratory, Parks Road, Oxford OX1 3PU, UK

3. School of Physics \& Astronomy, University of Nottingham, Nottingham, NG7 2RD, UK

*Correspondence to: harry.anderson@chem.ox.ac.uk, laura.herz@physics.ox.ac.uk or alex.saywell@nottingham.ac.uk

Natural light harvesting systems absorb sunlight and transfer its energy to the reaction centre, where it is used for photosynthesis. Synthetic chromophore arrays provide useful models for understanding energy migration in these systems. Research has focussed on mimicking rings of chlorophyll molecules found in purple bacteria, known as 'light-harvesting system 2'. Linear meso-meso linked porphyrin chains mediate rapid energy migration, but until now it has not been possible to bend them into rings. Here we show that oligo-pyridyl templates can be used to bend these rod-like photonic wires, to create covalent nanorings. The macrocycles consist of 24 porphyrin units, with a single butadiyne link. Their elliptical conformations have been probed by scanning tunnelling microscopy (STM). This system exhibits two types of excited state energy transfer processes: (a) from the central template to the peripheral porphyrins and (b) from the 24 light-absorbing porphyrin units to the $\pi$-conjugated butadiyne-linked porphyrin dimer segment.

Green plants, and other photosynthetic organisms, capture sunlight using antenna complexes that consist of large arrays of chlorophyll molecules. Electronic excitation is funnelled through the antenna complex into a reaction centre, where it is converted into chemical energy ${ }^{1,2}$. There are typically 100-800 light-absorbing chlorophyll units per reaction centre ${ }^{3}$, and each absorption event results in a long cascade of electronic excitation 
energy transfer (EET) steps. Light harvesting systems have evolved so that this EET process is extremely rapid, and it occurs efficiently without significant competition from other decay channels, such as fluorescence, intersystem crossing and non-radiative internal conversion. Different organisms use a wide variety of light-harvesting chlorophyll antenna complexes. Two of the most highly studied examples are light-harvesting systems 1 and 2 (LH1 and LH2) from purple bacteria ${ }^{1}$. LH2 consists of two concentric rings of bacteriochlorophyll units (both with diameters of $\sim 6 \mathrm{~nm})^{4}$ : the B800 ring of 9 chlorophylls and the B850 ring of 18 chlorophylls. The B850 ring is remarkable because its 18 bacteriochlorophyll units are closely spaced and strongly coupled, resulting in ultra-fast intra-ring EET $(<200 \mathrm{fs})^{2}$. Many rings of porphyrin units have been synthesised and investigated as models for $\mathrm{LH}^{5-12}$, but in most cases the EET in these models is much slower than in the natural B850 ring. The only synthetic cyclic porphyrin arrays that mimic intra-ring EET on the same time scale $(<200 \mathrm{fs})$ and length scale ( $\sim 6 \mathrm{~nm}$ diameter) as the B850 ring are the butadiyne-linked porphyrin nanorings developed in our laboratories ${ }^{8,9}$. However, these rings achieve strong porphyrinporphyrin coupling through $\pi$-conjugation (i.e. direct orbital overlap), which makes them fundamentally different from the exciton-coupled rings of chlorophyll units in LH2. In this paper, we explore the synthesis and photophysical properties of rings consisting of closely spaced exciton-coupled porphyrin units, with diameters of about $6 \mathrm{~nm}$, which exhibit ultrafast EET.

Kim, Osuka and coworkers have shown that directly meso-meso linked linear porphyrin arrays (l-PN, Figure 1) behave as 'photonic wires' and exhibit ultrafast EET due to strong exciton coupling between neighbouring porphyrin units, with radiative coherent lengths of about 6 porphyrin units ${ }^{13,14}$. The corresponding cyclic 5,15 -linked porphyrin nanorings ( $c$ PN, Figure 1) would be fascinating models for LH2, but it would be difficult to study EET in these highly symmetric rings because there would be no directional energy flow. It would be easier to probe EET in structures such as $\boldsymbol{c}-\mathbf{P} \mathbf{N} \mathbf{b}$, with a single butadiyne link, because excitation would then migrate to the site of the butadiyne, because a $\pi$-conjugated butadiynelinked porphyrin dimer has a lower $\mathrm{S}_{1}$ excited state than a meso-meso linked porphyrin oligomer. The single butadiyne link in $\boldsymbol{c}-\mathbf{P} \mathbf{N} \mathbf{b}$ also makes it easier to synthesise. Here we report the template-directed synthesis of a 24-porphyrin nanoring of this type with a single butadiyne link. Oligomers of the type $\boldsymbol{l}-\mathbf{P} \boldsymbol{N}$ are often described as "rodlike"13, which raises the question of whether we can bend a straight rod into a ring. Here we show that molecular templates can be used to enforce a circular geometry to create this strained nanoring. 


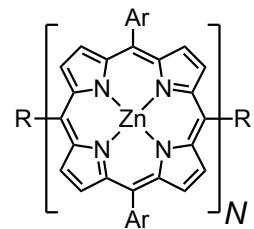

I-PN $(\mathrm{R}=\mathrm{H})$

I-PNe $(\mathrm{R}=\mathrm{C} \equiv \mathrm{CH})$
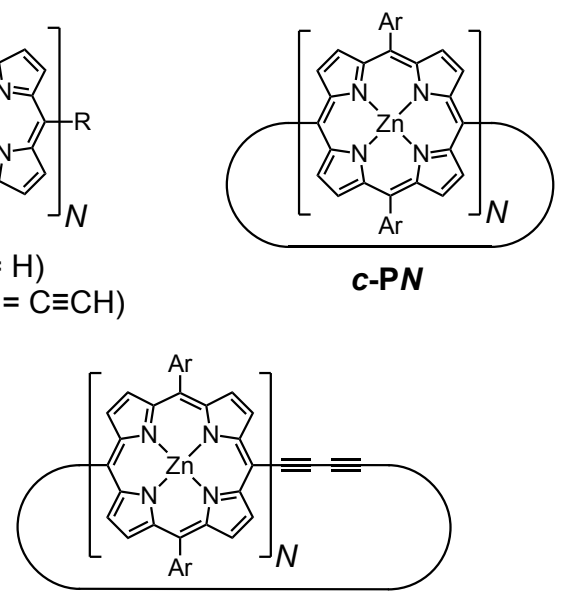

$c-\mathrm{PNb}$

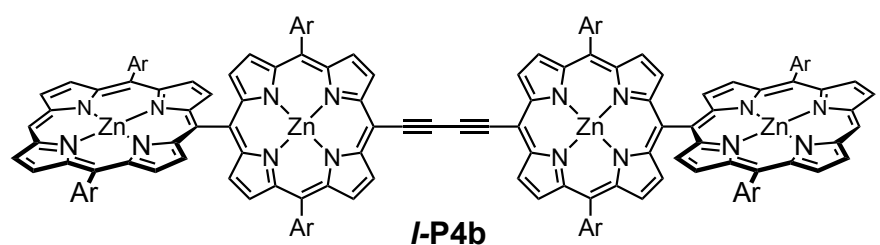

Fig. 1 | Structures of porphyrin oligomers, $\boldsymbol{l}-\mathbf{P} N, \boldsymbol{l}-\mathbf{P N e}, \boldsymbol{c}-\mathbf{P} \boldsymbol{N}$ and $\boldsymbol{c}-\mathbf{P N b}$ (where $N$ is the number of porphyrin units; $\boldsymbol{l}-, \boldsymbol{c}$-, $\mathbf{e}$ and $\mathbf{b}$ indicate linear, cyclic, ethynyl and butadiyne, respectively), and the reference compound $\boldsymbol{l}$-P4b. Ar is the 3,5-bis(octyloxy)phenyl solubilising group.

\section{Results and Discussion}

Computational modelling and design of the template. We started this project by calculating the strain in $\boldsymbol{c}-\mathbf{P} \boldsymbol{N}$ and $\boldsymbol{c}-\mathbf{P N b}$, as a function $N$, by considering gas-phase homodesmotic reactions, using both molecular mechanics calculations and density functional theory (DFT) (see Supplementary Information for details). As expected, the level of strain is high in the smaller macrocycles (e.g. $\boldsymbol{c}-\mathbf{P 1 2}$ : $155.8 \mathrm{~kJ} \mathrm{~mol}^{-1}$ and $\boldsymbol{c}-\mathbf{P 1 2 b}$ : $143.5 \mathrm{~kJ} \mathrm{~mol}^{-1}$ ), but declines as the rings become larger. Thus the strain energies of $\boldsymbol{c}$-P24 $\left(78.7 \mathrm{~kJ} \mathrm{~mol}^{-1}\right)$ or $\boldsymbol{c}$-P24b $\left(75.7 \mathrm{~kJ} \mathrm{~mol}^{-1}\right)$ are less than in the alkyne-linked porphyrin nanorings that we have reported previously $\left(100-130 \mathrm{~kJ} \mathrm{~mol}^{-1}\right)^{15}$, implying that they are reasonable targets for template-directed synthesis.

One challenge in designing a template to bind a linear oligo-porphyrin, such as $\boldsymbol{l}$ $\mathbf{P 2 4}$, to direct the formation of $\mathbf{c - P 2 4 b}$, is that meso-meso singly-linked oligomers have twisted conformations ${ }^{16,17}$. The dihedral angle between neighbouring zinc porphyrins is typically $70-90^{\circ}$, so that the axial vectors of the porphyrin units in $\mathbf{c}-\mathbf{P} 24 \mathbf{b}$ do not point towards the centre of the nanoring. Inspired by the work of Osuka and coworkers ${ }^{16}$ on the binding of $\alpha, \omega$-diaminoalkanes to dimers such as $\boldsymbol{l}$-P2, we designed a binding unit $\mathbf{L}$, 
which has two 4-pyridyl binding sites connected to a benzene core via flexible metalinked $-\mathrm{O}\left(\mathrm{CH}_{2}\right)_{3}-$ chains. Computational studies indicated that this ligand would coordinate the two zinc centres of the twisted porphyrin dimer $\boldsymbol{l}$-P2 and this was confirmed by UV-vis binding studies, which gave a binding constant $7.8 \times 10^{5} \mathrm{M}^{-1}$ (in $\mathrm{CDCl}_{3}$ at 298 $\mathrm{K})$, compared with $1.2 \times 10^{4} \mathrm{M}^{-1}$ for the $\mathbf{P 1}$ monomer binding 4-ethyl pyridine, under identical conditions. Although the effective molarity of this system is quite low $(E M=2.7$ $\mathrm{mM}$ ), it is high enough for the $\boldsymbol{l}$-P2 $\cdot \mathbf{L}$ complex to be $94 \%$ closed (i.e. coordinated at both sites $)^{18}$.

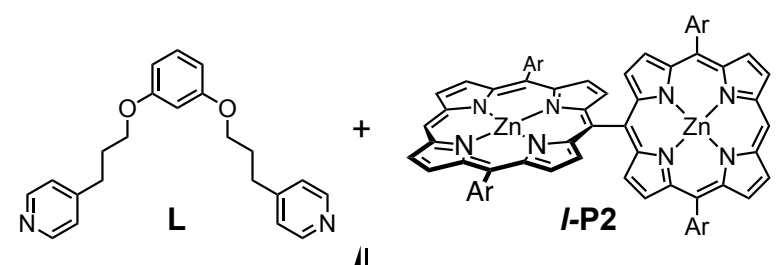

॥ $K_{\mathrm{f}}=7.8 \times 10^{5} \mathrm{M}^{-1}$

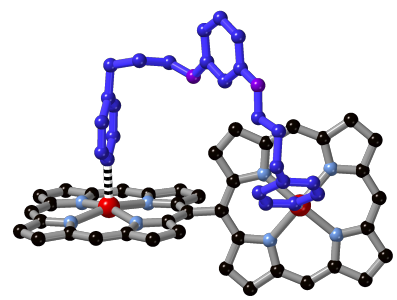

Fig. 2 Chelation of the binding unit $\mathbf{L}$ to l-P2. The binding constant $\left(7.8 \times 10^{5} \mathrm{M}^{-1}\right)$ was measured by UV-vis titration in $\mathrm{CDCl}_{3}$ at $298 \mathrm{~K}$ and compares with a value of $1.2 \times$ $10^{4} \mathrm{M}^{-1}$ for the corresponding P1 monomer binding 4-ethyl pyridine. The optimised geometry of $\boldsymbol{l}$-P2 $\cdot \mathbf{L}$ was calculated using DFT (PBE0+GD3BJ/Def2SVP). Ar $=3,5-$ bis(octyloxy)phenyl.

It would be difficult to design a single template capable of binding all 24 zinc sites in l-P24e, to bend it into a circular conformation. Instead, we designed the T12 template, which has six $\mathbf{L}$ binding units linked to a benzene core, and is shaped so that a cofacial stack of two molecules of $\mathbf{T 1 2}$ can bind inside the cavity of $\boldsymbol{c}$-P24b (Figure 3) ${ }^{19}$. Molecular dynamics simulations of $l$-P24e·(T12) 2 predicted that all 24 of the pyridyl binding sites of the templates remain coordinated to zinc atoms in the 1:2 complex. General AMBER force field ${ }^{20}$ parameters were employed together with novel hybrid bonded/non-bonded parameters for the zinc ions that were adjusted to reproduce the coordination geometry and energy of pyridine zinc interactions. The $l$-P24e-(T12) complex is predicted to exist as two isomers with similar energies (binding modes A and $\mathrm{B}$, Figure 4). The angle $\theta$ and distance $d$ between the terminal alkynes fluctuates across a 
distribution of values in these complexes (Figure 4b,c), including those in a range suitable for Glaser coupling ${ }^{21}$. There is no strong correlation between the two parameters, $\theta$ and $d$, because the alkynes tend to move apart by displacement away from the mean plane of the complex, rather than unwrapping from the templates.

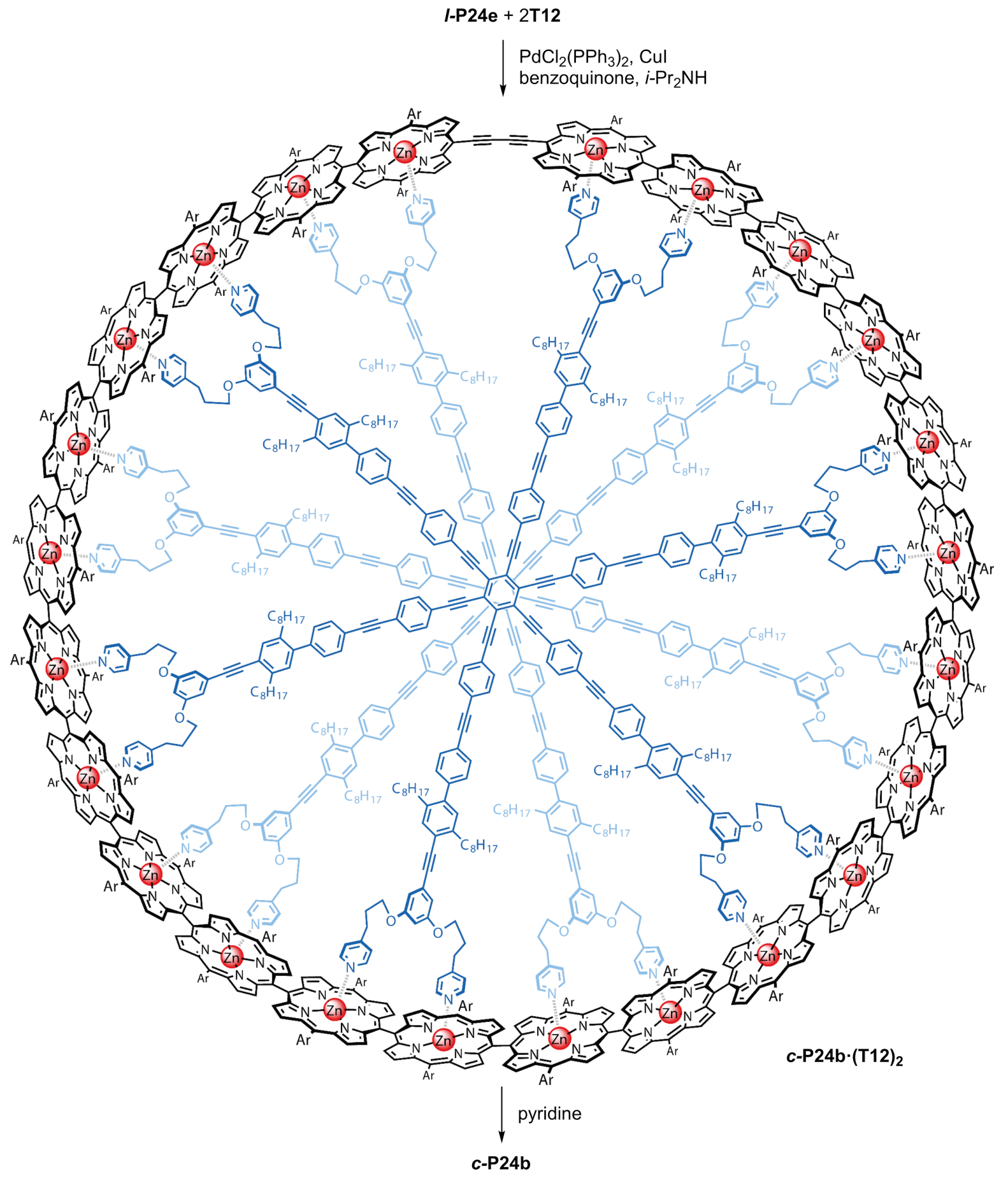

Fig. 3 | Reaction scheme showing the synthesis of $c$-P24b via the template complex $c$ P24b·(T12)2. The solubilising side chains on all the porphyrins are $\mathrm{Ar}=3,5-$ bis(octyloxy)phenyl. 
a

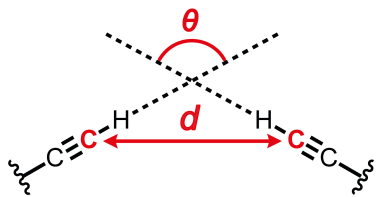

b
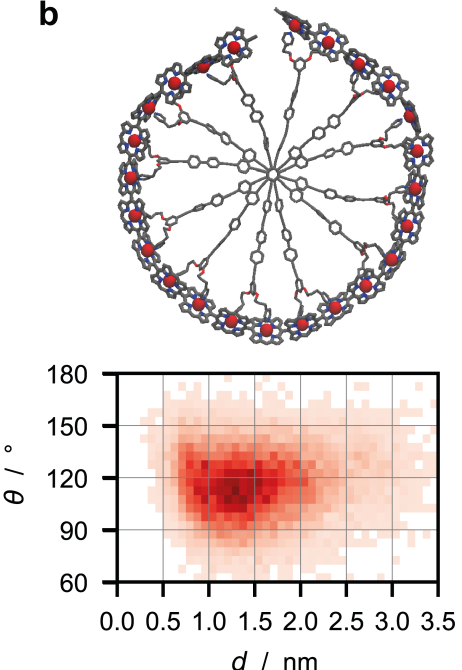

I-P24e (T12) mode $A$
C
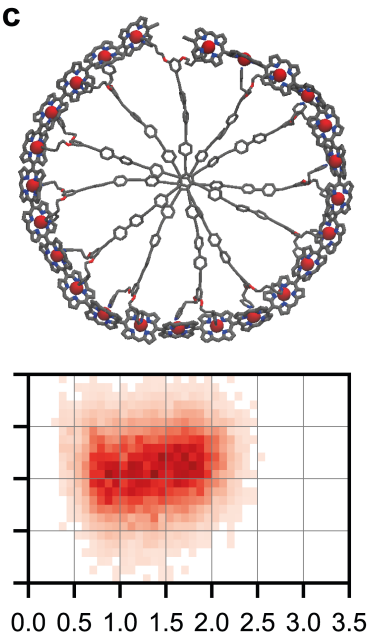

d / $\mathrm{nm}$

l-P24e•(T12) mode $B$

Fig. 4 | Molecular dynamics simulations of $\boldsymbol{l}$-P24e·(T12)2. a, Definition of parameters $d$ and $\theta$. $\mathbf{b}$ and $\mathbf{c}$, Geometry distributions for the two binding modes $\mathrm{A}$ and $\mathrm{B}$ with structures representative of the highest probabilities of $d$ and $\theta$.

Synthesis and chemical characterisation. Palladium-catalysed oxidative coupling of $\boldsymbol{l}$ P24e in the presence of T12, followed by displacement of the template with pyridine, gave nanoring $\boldsymbol{c - P 2 4 b}$ in $26 \%$ yield (Figure 3 ). This compound was initially identified from its retention time by gel-permeation chromatography (GPC) and by observation of its molecular ion in mass spectrometry (found $m / z 24,927.5$; calc. $\mathrm{C}_{1540} \mathrm{H}_{1968} \mathrm{~N}_{96} \mathrm{O}_{96} \mathrm{Zn}_{24}$ : 24,930.0). The GPC retention time of $\boldsymbol{c}$-P24b is $4.6 \%$ longer than that of $\boldsymbol{l}$-P24e, as expected for the smaller hydrodynamic radius of the cyclic compound ${ }^{22}$. The same effect is seen by diffusion-ordered NMR spectroscopy (DOSY): $\boldsymbol{c}$-P24b and $\boldsymbol{l}$-P24 have diffusion coefficients of $1.37 \times 10^{-10} \mathrm{~m}^{2} \mathrm{~s}^{-1}$ and $9.40 \times 10^{-11} \mathrm{~m}^{2} \mathrm{~s}^{-1}$, respectively, in $\mathrm{CDCl}_{3}$ at $298 \mathrm{~K}$. The ${ }^{1} \mathrm{H}$ NMR spectrum of $\boldsymbol{c}$-P24b is similar to that of $\boldsymbol{l}$-P24e, except without the terminal alkyne peak at $\delta_{\mathrm{H}} 4.28 \mathrm{ppm}$. The many similar unresolved porphyrin ${ }^{1} \mathrm{H}$ NMR environments of the cyclic compound also show a wider chemical shift dispersion than for the linear chain. 
The interaction of the nanoring $\boldsymbol{c}$-P24b with template $\mathbf{T 1 2}$ was studied by UV-vis and fluorescence titrations (in $\mathrm{CDCl}_{3}$ at $298 \mathrm{~K}$ ). The formation titration confirms that $c$ $\mathbf{P 2 4 b}$ binds strongly with two equivalents of $\mathbf{T 1 2}$ to give a 1:2 complex $\boldsymbol{c}-\mathbf{P 2 4 b} \cdot(\mathbf{T 1 2})_{2}$ (Figures S20 and S21). The formation constant $\left(K_{\mathrm{f}}\right)$ is too high to measure directly from the formation titration, and denaturation titrations, in which a competing ligand (quinuclidine) is added to displace the template, were used to determine the stability of the complex $^{23}$. Displacement of $\mathbf{T 1 2}$ from $\boldsymbol{c}-\mathbf{P 2 4 b} \cdot(\mathbf{T 1 2})_{2}$ with increasing quinuclidine concentration can be monitored by the turn-on response of fluorescence at $472 \mathrm{~nm}$, because the free $\mathbf{T 1 2}$ template is highly florescent, whereas its fluorescence is quenched in the complex (due to energy transfer to the porphyrin nanoring, as shown below). Analysis of the binding isotherms shows that the 1:2 complex $\boldsymbol{c}-\mathbf{P 2 4 b} \cdot(\mathbf{T 1 2})_{2}$ is extremely stable with $\log \left(K_{\mathrm{f}}\right)=81.5 \pm 0.7, \Delta G=465 \mathrm{~kJ} \mathrm{~mol}^{-1}$, which illustrates that there is ample binding energy to overcome the strain energy required to form the nanoring.

We also attempted to synthesise a smaller nanoring $\boldsymbol{c}$-P12b using two smaller versions of T12, but no cyclic products were formed from $\mathbf{l}$-P12e in either case. This can probably be attributed to the high strain in $\mathbf{c - P 1 2 b}$ (see Supplementary Information, Section 4 and Figure S5).

Scanning probe microscopy. The $c$-P24b nanoring was transferred from solution in toluene/methanol onto a $\mathrm{Au}(111)$ substrate, held under vacuum conditions, by electrospray deposition using a previously reported procedure ${ }^{24}$. Images of the molecules were recorded by scanning tunnelling microscopy (STM), as illustrated in Figure 5a,b (and Figures S12S15). The variation in contrast around the nanorings is attributed to the non-planar orientation of the porphyrin sub-units, with the brighter features assigned to porphyrins tilted upwards from the surface plane. The measured long and short axes of the rings indicate some structural flexibility. Average values of the long axis $(a=6.9 \mathrm{~nm}$, SD 0.9 $\mathrm{nm})$ and short axis $(b=4.3 \mathrm{~nm}, \mathrm{SD} 0.7 \mathrm{~nm})$ correspond to an ellipticity or flattening factor of $f=1-b / a=0.4(\mathrm{SD}=0.2)$ on the gold surface, which is significantly greater than for the optimised geometry from DFT ( $a=6.8 \mathrm{~nm} ; b=6.4 \mathrm{~nm} ; f=0.07$; Figure $5 \mathrm{c}$ ). The distribution of conformations observed by STM is compared with that from molecular dynamics calculations (at $300 \mathrm{~K}$ with explicit $\mathrm{CHCl}_{3}$ solvent; $a=6.9 \mathrm{~nm}$, $\mathrm{SD} 0.3 \mathrm{~nm} ; b=$ $5.8 \mathrm{~nm}, \mathrm{SD} 0.3 \mathrm{~nm} ; f=0.16$, SD 0.07$)$ in Figure $5 \mathrm{~d}$. The mean circumference from the molecular dynamics calculations $(c=20.0 \mathrm{~nm})$ is slightly larger than the apparent average 
circumference from the STM images $(18.2 \mathrm{~nm}$, for the average of 38 images), but this probably reflects the fact that the molecules on the surface are not exactly elliptical.
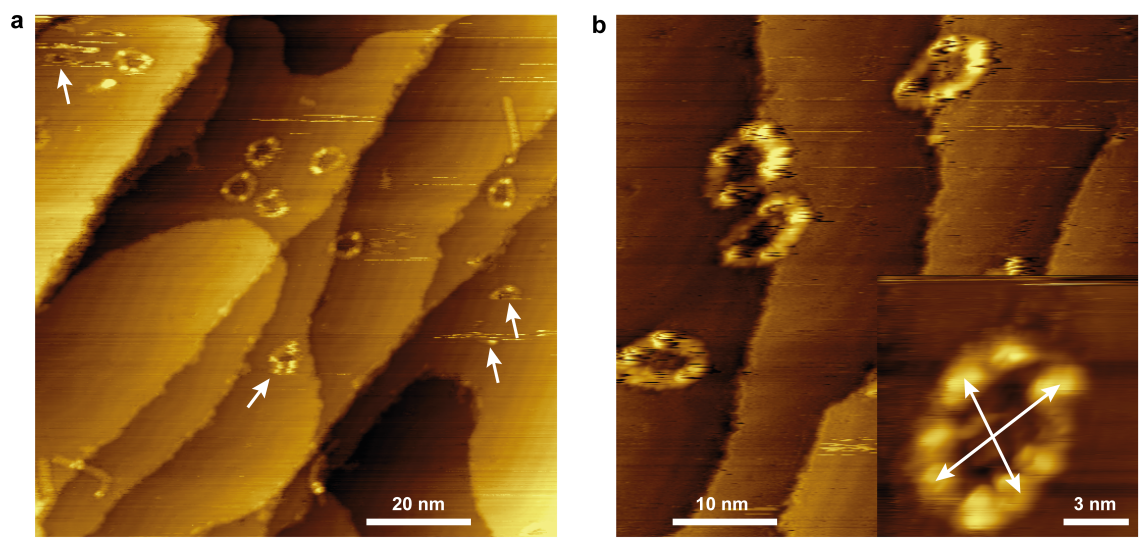

c

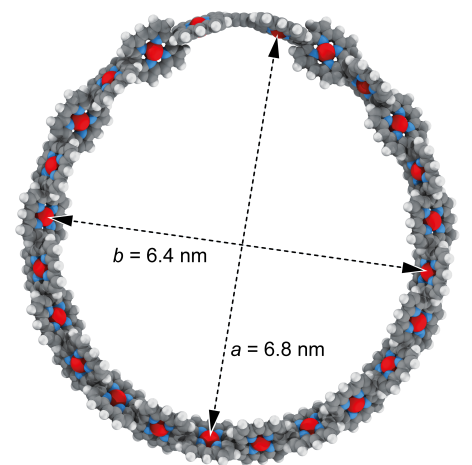

d

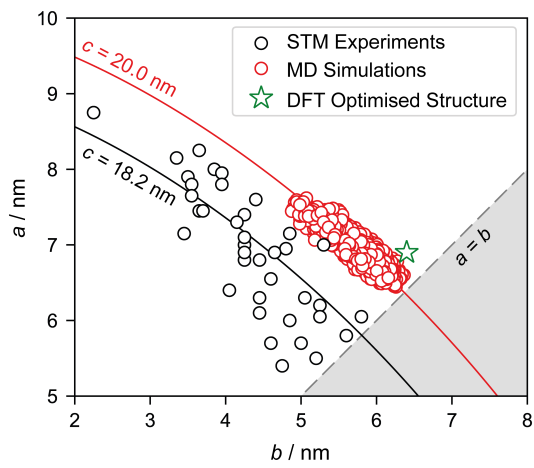

Fig. 5 | STM characterisation of $\boldsymbol{c}$-P24b on Au(111). a-b, STM-images of the $\boldsymbol{c}$-P24b nanoring deposited on a Au(111) surface under UHV (-1.8 V sample-bias, 20 pA set-point current, $T=293 \mathrm{~K}$, image sizes; $100 \times 100 \mathrm{~nm}, 50 \times 50 \mathrm{~nm}$, and $12 \times 12 \mathrm{~nm}$ ). In panel (a), arrows indicate $\boldsymbol{c} \mathbf{- P 2 4 b}$ nanorings which are mobile on the timescale of image acquisition. In panel (b), arrows show an example of the measured dimensions. c, Optimised geometry of $\boldsymbol{c}$-P24b (PBE0+GD3BJ/Def2SVP). Aryl and octyl solubilising groups were replaced by hydrogen to simplify the calculations. d, plot of long $(a)$ vs. short $(b)$ axes values for 38 rings observed by STM (black circles) and points from the molecular dynamics simulation (red circles). The black and red curves are the corresponding best fit lines for ellipses of fixed circumference ( $c=18.2 \mathrm{~nm}$ and $20.0 \mathrm{~nm}$, respectively, calculated using Ramanujan's approximation). The dashed line indicates circular geometries, $a=b$.

Interactions with substrate features, such as step-edges and the herringbone reconstruction of $\mathrm{Au}(111)$, may favour alternative conformations to those predicted by solution-phase calculations. The nanorings display significant mobility under these measurement conditions, with several lateral translation events observed (diffusion occurs at a faster rate than the acquisition time of an STM image - translation events highlighted with arrows in Figure 5a). This mobility indicates a decreased adsorption energy compared to the butadiyne-linked variant, which can be attributed to the nonplanarity of $\mathbf{c}-\mathbf{P} \mathbf{2 4 b}$. 
Photophysics and excited-state energy transfer. The UV-visible absorption and fluorescence spectra of $\boldsymbol{c - P 2 4 b}$ and $\boldsymbol{l}$-P24 are compared with those of a porphyrin tetramer with a central butadiyne link, $\mathbf{l}-\mathbf{P} \mathbf{4 b}$, in Figure $6 \mathrm{a}, \mathrm{b}$. The absorption spectra of $\boldsymbol{c}-\mathbf{P 2 4 b}$ and l-P24 feature intense bands at 419, 515 and $594 \mathrm{~nm}$ which are assigned to the $\mathrm{B}_{\mathrm{y}}, \mathrm{B}_{\mathrm{x}}$ and $\mathrm{Q}$ transition, respectively ${ }^{13}$. The absorption spectra of $\boldsymbol{c}$-P24b and $\boldsymbol{l}$-P4b also feature a weaker band at $650-700 \mathrm{~nm}$, which is not present for $\mathbf{l - P 2 4}$ and which is attributed to the $\pi$-conjugated butadiyne-linked porphyrin dimer unit (Figure 6a, insert). The fluorescence spectra of $\boldsymbol{c}$-P24b and $\boldsymbol{l}$-P4b are very similar with peaks at 729 and $723 \mathrm{~nm}$ respectively (Figure $6 \mathrm{~b})$, and both compounds emit at a longer wavelength than $\mathbf{l - P 2 4}\left(\lambda_{\max } 651 \mathrm{~nm}\right)$, which indicates that EET to the butadiyne-linked porphyrin dimer unit occurs efficiently in $\boldsymbol{c}$-P24b. Comparison of the steady-state emission spectra of $\boldsymbol{l}-\mathbf{P 2 4}, \boldsymbol{l}-\mathbf{P} 4 \mathbf{b}$ and $\boldsymbol{c}-\mathbf{P 2 4 b}$ (Figure 6b) implies that the EET efficiency is $>95 \%$.

The EET process in $\boldsymbol{c}$-P24b was investigated by monitoring the evolution of its fluorescence on a ps-timescale through fluorescence up-conversion spectroscopy (time resolution: $\sim 350 \mathrm{fs}$ ). The fluorescence from $\boldsymbol{c}$-P24b displays a rapid decay at $650 \mathrm{~nm}$ and a concomitant rise at $720 \mathrm{~nm}$ (Figures 6c, S33 and S34). A stretched exponential decay, equation (1), provides an excellent description of the $650 \mathrm{~nm}$ emission kinetics for the ring (see Supplementary Information, Section 9). ${ }^{25}$

$$
I(t)=I_{0} \exp \left[-\left(t / \tau_{0}\right)^{\beta}\right]
$$

where $I(t)$ is the fluorescence intensity, $t$ is time, $\tau_{0}$ is the time constant and $\beta$ is the stretching exponent. The extracted parameters are $\tau_{0}=8.5 \pm 0.6$ ps and $\beta=0.54 \pm 0.04$. The stretching exponent is significantly below unity (monoexponential decay), indicating that a range of excitation transfer times is present, as expected for random placement of excitation across the different porphyrins units comprising the ring. The characteristic transfer time $\tau_{0}$ represents an average time taken for an excitation to migrate from porphyrin donors to the butadiyne acceptor unit. The matching rise dynamics observed in the emission at $720 \mathrm{~nm}$ confirms that these coupled dynamics are associated with energy transfer. Modelling of the transient fluorescence at 650 and $720 \mathrm{~nm}$ (Figure 6c, S34) with an exciton generation function (Supplementary Information, equation S13) indicates that 
approximately $40 \%$ of the excitation arrives at the butadiyne-linked porphyrin unit within the time-resolution. We estimate from the absorption spectra (Figure $6 a$ ) that $9 \%$ of the
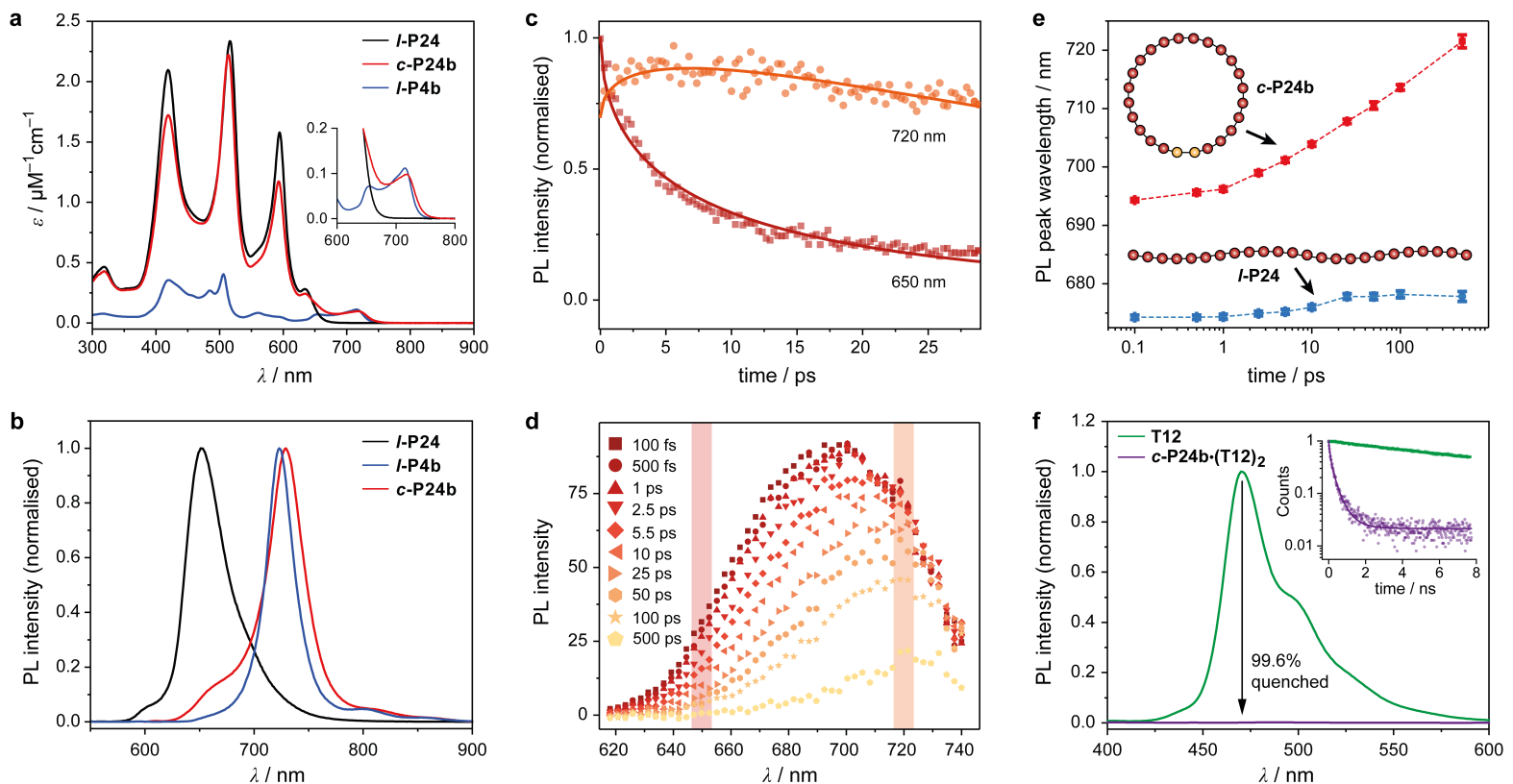

Fig. 6 | Absorption and fluorescence spectra. a, UV-vis absorption spectra. b, Steadystate fluorescence spectra. c, Transient fluorescence of $\boldsymbol{c - P 2 4 b}$, detected at $650 \mathrm{~nm}$ (red squares) and $720 \mathrm{~nm}$ (orange circles) under $410 \mathrm{~nm}$ wavelength excitation at $2.5 \mu \mathrm{J} \mathrm{cm}{ }^{-2}$. Solid lines represent fits to the model described in Supplementary Information, Section 9. d, Time-resolved fluorescence spectra for $\mathbf{c - P 2 4 b}$ following 410-nm wavelength pulsed excitation at a fluence of $2.5 \mu \mathrm{J} \mathrm{cm}^{-2}$. Different symbols represent spectra taken at different times after the excitation, in the range $100 \mathrm{fs}$ (dark red square) - 500 ps (yellow pentagon). e, Comparison between the time evolution of the peak wavelength of the photoluminescence spectra for $\boldsymbol{c}$-P24b (red squares), and $\boldsymbol{l}$-P24 (blue circles). Data points are extracted through Gaussian fitting of the time-resolved photoluminescence spectra measured under the same excitation as a function of time after excitation. Dashed lines are guides for the eye. f, Emission spectra of T12 $\left(1.6 \times 10^{-7} \mathrm{M}\right)$ and $\boldsymbol{c}$-P24b $\cdot(\mathbf{T 1 2})_{2}$ $\left(0.8 \times 10^{-8} \mathrm{M}\right)$, i.e. at identical template concentration, with excitation at $318 \mathrm{~nm}$. The T12 quenching efficiency in the complex is $99.6 \%$. The insert shows the transient fluorescence decay from both solutions (excitation wavelength: $380 \mathrm{~nm}$, power $6 \mathrm{~mW}$ ). The free template exhibits a mono-exponential decay $\tau=8 \pm 1 \mathrm{~ns}$. The template emission can be fitted with stretched exponential decay $\left(\tau_{0}=0.15 \pm 0.04 \mathrm{~ns} ; \beta=0.7\right)$. All spectra were recorded in toluene containing $1 \%$ pyridine at $298 \mathrm{~K}$, except for $\boldsymbol{c}$-P24b·(T12) which was dissolved in toluene without pyridine.

absorption at $410 \mathrm{~nm}$ is direct excitation of the butadiyne-linked acceptor site, which implies that about $30 \%$ of the EET occurs within the time resolution (350 fs). Further evidence of EET is provided by the pronounced redshift of the peak emission wavelength in $\boldsymbol{c - P 2 4 b}$, during 0-50 ps after excitation (Figure 6d,e). Again, it is clear that part of the 
EET occurs within the time resolution, because the emission maximum of $\boldsymbol{c}$-P24b is redshifted with respect to that of $\mathbf{l}$-P24 even at the earliest times we can measure (Figure $6 \mathrm{e})$. On a longer timescale ( $\sim 500 \mathrm{ps}$ ), the emission spectrum of $\boldsymbol{c}$-P24b exhibits a slower redshift due to planarisation of the excited state of the butadiyne-linked porphyrin dimer unit $^{26,27}$; this slow process is also observed in $\boldsymbol{l}$-P4b. In contrast, $\boldsymbol{l}$-P24 displays only weak shifts due to EET among segments of the chain with different porphyrin-porphyrin dihedral angles. ${ }^{13}$ It is interesting that the average rate of EET in $\boldsymbol{c}$-P24b is significantly faster than in a similar linear 24-porphyrin chain terminated with an ethynyl-porphyrin energy acceptor (time constant $110 \mathrm{ps})^{28,29}$.

Energy transfer is also observed from the central template units in $\boldsymbol{c}-\mathbf{P 2 4 b} \cdot(\mathbf{T 1 2})_{2}$ to the surrounding zinc porphyrins. The T12 template is highly fluorescent $\left(\lambda_{\mathrm{em}}: 472 \mathrm{~nm}\right.$; $\phi_{\mathrm{f}}=$ 0.26 , in toluene at $298 \mathrm{~K}$ ) and its fluorescence is quenched (by a factor of $99.6 \%$ ) in the $c$ P24b·(T12) 2 complex, as mentioned above and shown in Figure 6f. The excitation spectrum of $\boldsymbol{c - P 2 4 b \cdot ( T 1 2 ) 2}$, based on emission at $760 \mathrm{~nm}$, has a peak at $319 \mathrm{~nm}$ corresponding to the absorption spectrum of the template (Figure S28), which demonstrates that there is energy transfer from the T12 unit to the porphyrin manifold. Analysis of transient fluorescence decays (inset of Figure 6f) reveals an average energy transfer time of $0.15 \mathrm{~ns}$.

\section{Conclusion}

This work demonstrates that oligo-pyridine templates can be used to direct the synthesis of large zinc porphyrin nanorings, even from linear oligomers that are twisted and rod-like. It also illustrates the power of molecular dynamics simulations to guide the design of templates. STM images confirm that the nanorings adopt elliptical conformations, similar to those predicted computationally. Excited state energy transfer from the 24-light absorbing zinc porphyrin units to the butadiyne-linked segment of $\boldsymbol{c}$-P24b has an efficiency $>95 \%$. This EET process occurs over a range of time scales, as expected from the distribution of donor-acceptor distances. About $30 \%$ of the energy is transferred within the time resolution of our measurements $(350 \mathrm{fs})$, while the EET process has an average time constant of 8.5 ps. The $\boldsymbol{c}$-P24b macrocycle mimics the photophysics of LH2, although the arrangement of transition dipoles in this nanoring, with neighbouring porphyrins almost orthogonal, seems to confer slower EET than the arrangement in the natural system, in which neighbouring chromophores are almost parallel. 


\section{Methods}

Synthesis of $\boldsymbol{c}$-P24b. A solution of T12 template $\left(5.3 \mathrm{mg}, 1.0 \mu \mathrm{mol}, 2.0\right.$ equiv) in $\mathrm{CHCl}_{3}$ $(220 \mathrm{~mL})$ was added to a solution of linear 24 -mer $\mathbf{l}$-P24e $(12.5 \mathrm{mg}, 0.50 \mu \mathrm{mol})$ in $\mathrm{CHCl}_{3}$ (250 mL). A catalyst mixture was prepared by dissolving CuI (54 mg, $284 \mu \mathrm{mol})$, $\mathrm{Pd}\left(\mathrm{PPh}_{3}\right)_{2} \mathrm{Cl}_{2}(39 \mathrm{mg}, 56 \mu \mathrm{mol})$, and 1,4-benzoquinone (121 mg, $\left.1120 \mu \mathrm{mol}\right)$ in DIPA (1.2 $\mathrm{mL})$ and $\mathrm{CHCl}_{3}(48 \mathrm{~mL})$ using sonication. The catalyst mixture was added to the reaction flask and stirred under air for $18 \mathrm{~h}$, while monitoring the reaction by analytical GPC. A solution of pyridine $(0.5 \mathrm{~mL})$ in THF $(50 \mathrm{~mL})$ was added to the reaction mixture and it was concentrated under reduced pressure to a volume of ca. $10 \mathrm{~mL}$, then passed through a short size-exclusion column (Bio-Beads S-X1, THF $+1 \%$ pyridine) to remove unreacted 1,4benzoquinone and copper salts. Purification by recycling GPC (loading in THF $+20 \%$ pyridine; eluting in $\mathrm{THF}+1 \%$ pyridine), followed by further size-exclusion chromatography (Bio-Beads S-X1, $\mathrm{CHCl}_{3}$ ) gave $\boldsymbol{c}$-P24b as a black solid (3.22 mg, 26\%). See Supplementary Information for characterisation data and related compounds.

Molecular Dynamics. All simulations were performed in an isothermal-isobaric (NPT) ensemble at $300 \mathrm{~K}$ and 1 bar with a time step of 2 fs using GROMACS (v. 2019.2). ${ }^{30}$ Systems were minimised using the steepest descent algorithm and subsequently equilibrated using a velocity-rescaling thermostat and Parrinello-Rahman barostat. All simulations were performed in explicit chloroform with three-dimensional periodic boundary conditions. See Supplementary Information for further details.

Scanning tunnelling microscopy (STM). Images were acquired with an Omicron STM-1 system operating under ultra-high vacuum (UHV) with a base pressure of $2 \times 10^{-9}$ mbar. c-P24b was transferred from solution (toluene/methanol) to a clean $\mathrm{Au}(111)$ substrate using electrospray deposition ${ }^{24,31}$. Images were acquired at room temperature in constant current mode using electrochemically etched tungsten tips, coated in gold during tip optimisation. All images taken at $-1.8 \mathrm{~V}$ sample-bias, $20 \mathrm{pA}$ set point current. See Supplementary Information for further details.

Optical spectroscopy. All measurements were carried out on solutions in toluene containing $1 \%$ pyridine (or neat toluene in the case of $\mathbf{T 1 2}$ and $\boldsymbol{c}-\mathbf{P 2 4 b} \cdot(\mathbf{T 1 2})_{2}$ ), in silica cuvettes of size $3.5 \times 10.0 \mathrm{~mm}$ or $10.0 \times 10.0 \mathrm{~mm}$, at a concentration of $0.25-2.0 \mu \mathrm{M}$ for steady-state measurements and $0.25-2.0 \mathrm{mM}$ for time-resolved experiments. Ultrafast time- 
resolved measurements were conducted using the photoluminescence upconversion (PL UC) technique. Samples were excited with a $410 \mathrm{~nm}$ pulse, generated by frequency doubling of a Ti:sapphire oscillator $820 \mathrm{~nm}$ output (Spectra Physics Mai-Tai, $100 \mathrm{fs}, 80$ $\mathrm{MHz}$ ). The side-excitation configuration was adopted to avoid possible artefacts caused by self-absorption. In this configuration, the excitation beam entered the cuvette close to its front surface and PL was collected in the perpendicular direction with respect to the excitation beam by a pair of off-axis parabolic mirrors. The PL signal was then focused onto a beta-barium borate (BBO) crystal, mounted on a rotation stage to allow tuning of the phase-matching angle, and optically gated by a vertically polarised gate beam ( 820 $\mathrm{nm}$ ) arriving at the BBO crystal at controllable time delays. The resulting PL UC signal generated by sum-frequency generation was then spectrally dispersed by a monochromator (Triax 190, Jobin-Yvon) and detected by a nitrogen-cooled CCD. A Schott filter UG11 was used to filter scattering from excitation and gate beams. The resulting time resolution was around 350 fs. Time-resolved spectra were corrected for instrument response by using a filament lamp of known emissivity. Time-integrated photoluminescence (TI PL) was measured by using the same spectrometer and $\mathrm{CCD}$ detector, while removing the $\mathrm{BBO}$ crystal and UG11 filter. Time-resolved PL dynamics at longer delay times ( $>1 \mathrm{~ns})$ were measured by using the time-correlated single-photon counting technique with a temporal resolution of around $40 \mathrm{ps}$.

\section{Data availability}

All relevant data, including raw computational data etc as well as XYZ coordinates of calculated molecular geometries, are available within the paper and its Supplementary Information files. The NMR and STM data are presented in detail in the main Supplementary Information file and are available upon reasonable request from the authors.

\section{References}

1. Cogdell, R. J., Gall, A. \& Köhler, J. The architecture and function of the lightharvesting apparatus of purple bacteria: from single molecules to in vivo membranes. Q. Rev. Biophys. 39, 227-324 (2006). 
2. Mirkovic, T., Ostroumov, E. E., Anna, J. M., van Grondelle, R., Govindjee \& Scholes, G. D. Light absorption and energy transfer in the antenna complexes of photosynthetic organisms. Chem. Rev. 117, 249-293 (2017).

3. Mauzerall, D. \& Greenbaum, N. L. The absolute size of a photosynthetic unit. Biochim. Biophys. Acta 974, 119-140 (1989).

4. McDermott, G., Prince, S. M., Freer, A. A., Hawthornthwaite-Lawless, A. M., Papiz, M. Z., Cogdell, R. J. \& Isaacs, N. W. Crystal structure of an integral membrane light-harvesting complex from photosynthetic bacteria. Nature 374, 517-521 (1995).

5. Cho, H. S., Rhee, H., Song, J. K., Min, C.-K., Takase, M., Aratani, N., Cho, S., Osuka, A., Joo, T. \& Kim, D. Excitation energy transport processes of porphyrin monomer, dimer, cyclic trimer, and hexamer probed by ultrafast fluorescence anisotropy decay. J. Am. Chem. Soc. 125, 5849-5860 (2003).

6. Choi, M.-S., Yamazaki, T., Yamazaki, I. \& Aida, T. Bioinspired molecular design of light-harvesting multiporphyrin arrays. Angew. Chem. Int. Ed. 43, 150-158 (2004).

7. Aratani, N., Kim, D. \& Osuka, A. Discrete cyclic porphyrin arrays as artificial lightharvesting antenna. Acc. Chem. Res. 42, 1922-1934 (2009).

8. Parkinson, P., Kondratuk, D. V., Menelaou, C., Gong, J. Q., Anderson, H. L. \& Herz, L. M. Chromophores in molecular nanorings: When is a ring a ring? J. Phys. Chem. Lett. 5, 4356-4361 (2014).

9. Yong, C.-K., Parkinson, P., Kondratuk, D. V., Chen, W.-H., Stannard, A., Summerfield, A., Sprafke, J. K., O'Sullivan, M. C., Beton, P. H., Anderson, H. L. \& Herz, L. M. Ultrafast delocalization of excitation in synthetic light-harvesting nanorings. Chem. Sci. 6, 181-189 (2015).

10. Otsuki, J. Supramolecular approach towards light-harvesting materials based on porphyrins and chlorophylls. J. Mater. Chem. A 6, 6710-6753 (2018).

11. Yang, J., Yoon, M.-C., Yoo, H., Kim, P. \& Kim, D. Excitation energy transfer in multiporphyrin arrays with cyclic architectures: towards artificial light-harvesting antenna complexes. Chem. Soc. Rev. 41, 4808-4826 (2012).

12. Aratani, N. \& Osuka, A. Exploration of giant functional porphyrin arrays. Bull. Chem. Soc. Jpn. 88, 1-27 (2015). 
13. Kim, Y. H., Jeong, D. H., Kim, D., Jeoung, S. C., Cho, H. S., Kim, S. K., Aratani, N. \& Osuka, A. Photophysical properties of long rodlike meso-meso-linked zinc(II) porphyrins investigated by time-resolved laser spectroscopic methods. J. Am. Chem. Soc. 123, 76-86 (2001).

14. Yang, J., Yoo, H., Aratani, N., Osuka, A. \& Kim, D. Determination of the superradiance coherence length of directly linked linear porphyrin arrays at the single-molecule level. Angew. Chem. Int. Ed. 48, 4323-4327 (2009).

15. Haver, R., Tejerina, L., Jiang, H.-W., Rickhaus, M., Jirasek, M., Grübner, I., Eggimann, H. J., Herz, L. M. \& Anderson, H. L. Tuning the circumference of sixporphyrin nanorings. J. Am. Chem. Soc. 141, 7965-7971 (2019).

16. Shinmori, H., Ahn, T. K., Cho, H. S., Kim, D., Yoshida, N. \& Osuka, A. Dihedralangle modulation of meso-meso-linked $\mathrm{Zn}^{\mathrm{II}}$ diporphyrin through diamine coordination and its application to reversible switching of excitation energy transfer. Angew. Chem. Int. Ed. 42, 2754-2758 (2003).

17. Yoshida, N., Ishizuka, T., Osuka, A., Jeong, D. H., Cho, H. S., Kim, D., Matsuzaki, Y., Nogami, A. \& Tanaka, K. Fine tuning of photophysical properties of meso-mesolinked $\mathrm{Zn}{ }^{\mathrm{II}}$-diporphyrins by dihedral angle control. Chem. Eur. J. 9, 58-75 (2003).

18. Hunter, C. A. \& Anderson, H. L. What is cooperativity? Angew. Chem. Int. Ed. 48, 7488-7499 (2009).

19. Bols, P. S.; Rickhaus, M., Tejerina, L.; Gotfredsen, H., Eriksen, K., Jirasek, M. \& Anderson, H. L. Allosteric cooperativity and template-directed synthesis with stacked ligands in porphyrin nanorings. J. Am. Chem. Soc. 142, 13219-13226 (2020).

20. Wang, J. M., Wolf, R. M., Caldwell, J. W., Kollman, P. A. \& Case, D. A. Development and testing of a general amber force field. J. Comput. Chem. 25, 11571174, (2004).

21. Fomina, L., Vazquez, B., Tkatchouk, E. \& Fomine, S. The Glaser reaction mechanism. A DFT study. Tetrahedron 58, 6741-6747 (2002).

22. Kondratuk, D. V., Perdigão, L. M. A., Esmail, A. M. S., O’Shea, J. N., Beton, P. H. \& Anderson, H. L. Supramolecular nesting of cyclic polymers. Nat. Chem. 7, 317$322(2015)$. 
23. Hogben, H. J., Sprafke, J. K., Hoffmann, M., Pawlicki, M., \& Anderson, H. L. Stepwise effective molarities in porphyrin oligomer complexes: preorganization results in exceptionally strong chelate cooperativity. J. Am. Chem. Soc. 133, 2096220969 (2011).

24. Judd, C. J.; Nizovtsev, A. S.; Plougmann, R.; Kondratuk, D. V.; Anderson, H. L.; Besley E. \& Saywell, A. Molecular quantum rings formed from a $\pi$-conjugated macrocycle. Phys. Rev. Lett. 125, 206803 (2020).

25. Berberan-Santos, M. N., Bodunov, E. N. \& Valeur, B. Mathematical functions for the analysis of luminescence decays with underlying distributions 1. Kohlrausch decay function (stretched exponential). Chem. Phys. 315, 171-182 (2005).

26. Winters, M. U., Kärnbratt, J., Eng, M., Wilson, C. J., Anderson, H. L. \& Albinsson, B. Photophysics of a butadiyne-linked porphyrin dimer: Influence of conformational flexibility in the ground and first singlet excited state. J. Phys. Chem. C 111, 71927199 (2007).

27. Chang, M.-H., Hoffmann, M., Anderson, H. L. \& Herz, L. M. Dynamics of excitedstate conformational relaxation and electronic delocalization in conjugated porphyrin oligomers. J. Am. Chem. Soc. 130, 10171-10178 (2008).

28. Aratani, N., Cho, H. S., Ahn, T. K., Cho, S., Kim, D., Sumi, H. \& Osuka, A. Efficient excitation energy transfer in long meso-meso linked $\mathrm{Zn}$ (II) porphyrin arrays bearing a 5,15-bisphenylethynylated Zn(II) porphyrin acceptor. J. Am. Chem. Soc. 125, 9668-9681 (2003).

29. Ahn, T. K., Yoon, Z. S., Hwang, I.-W., Lim, J. K., Rhee, H., Joo, T., Sim, E., Kim, S. K., Aratani, N., Osuka, A. \& Kim, D. Effect of conformational heterogeneity on excitation energy transfer efficiency in directly meso-meso linked $\mathrm{Zn}(\mathrm{II})$ porphyrin arrays. J. Phys. Chem. B 109, 11223-11230 (2005).

30. Abraham, M. J., Murtola, T., Schulz, R., Páll, S., Smith, J. C., Hess, B. \& Lindahl, E. GROMACS: High performance molecular simulations through multi-level parallelism from laptops to supercomputers. SoftwareX 1-2, 19-25, (2015).

31. Judd, C. J.; Kondratuk, D. V.; Anderson, H. L. \& Saywell, A. On-surface synthesis within a porphyrin nanoring template. Sci. Rep. 9, 9352 (2019). 
Acknowledgments. We thank the ERC (grant 885606, ARO-MAT) for funding. H.G. thanks the Independent Research Fund Denmark for an International Postdoctoral Fellowship. A.S. thanks the Royal Society for support via a University Research Fellowship. Computational services were provided by the Advanced Research Computing Service at the University of Oxford. M.R. and L.M.H. acknowledge funding by the Engineering and Physical Sciences Research Council UK. L.M.H. acknowledges support through a Hans Fischer Senior Fellowship from the Technical University of Munich's Institute for Advanced Study, funded by the German Excellence Initiative.

\section{Author contributions}

H.G., J.-R.D. and J.V.R. synthesised and characterised the compounds. J.H. and F.D. carried out the computational modelling, after preliminary modelling by H.G., J.-R.D. and J.V.R.; T.D.W.C. assisted with NMR experiments; A.B.-C., M.C. and A.S. performed the scanning probe microscopy. J.H. and J.O. prepared samples via electrospray deposition. M.R. and L.M.H. investigated the time-resolved photophysics. H.L.A. and H.G. wrote the paper; all authors discussed the results and edited the manuscript.

\section{Competing interests}

The authors declare no competing interests.

\section{Additional information}

Supplementary information is available for this paper at https://doi.org/10.?????

\section{Graphical Abstract:}

